\title{
Research and Ground Verification of the Force Compliance Control Method for Space Station Manipulator
}

\author{
Bingshan Hu $\mathbb{D},{ }^{1}$ Huanlong Chen, ${ }^{2}$ Liangliang Han, ${ }^{2}$ and Hongliu $\mathrm{Yu}^{1}$ \\ ${ }^{1}$ University of Shanghai for Science and Technology, Shanghai 200093, China \\ ${ }^{2}$ Shanghai Institute of Aerospace Systems Engineering, Shanghai 201109, China \\ Correspondence should be addressed to Bingshan Hu; icebergh@126.com
}

Received 20 April 2020; Revised 17 August 2020; Accepted 20 August 2020; Published 3 September 2020

Academic Editor: Giovanni Palmerini

Copyright (C) 2020 Bingshan $\mathrm{Hu}$ et al. This is an open access article distributed under the Creative Commons Attribution License, which permits unrestricted use, distribution, and reproduction in any medium, provided the original work is properly cited.

\begin{abstract}
The space station manipulator does lots of tasks with contact force/torque on orbit. To ensure the safety of the space station and the manipulator, the contact force/torque of manipulator must be controlled. Based on analyzing typical tasks' working flows and force control requirements, such as ORU (orbit replacement unit) changeout and dual arm collaborative payload transport, an impedance control method based on wrist 6 axis force/torque feedback is designed. For engineering implementation of the impedance control algorithm, the discretization method and impedance control parameters selection principle are also studied. To verify the compliance control algorithm, a ground experiment platform adopting industrial manipulators is developed. In order to eliminate the influence of gravity, a real-time gravity compensation algorithm is proposed. Then, the correctness of real-time gravity compensation and force compliance control algorithm is verified on the experiment platform. Finally, the ORU replacement and dual arm collaborative payload transport experiments are done. Experimental results show that the force compliance control method proposed in this paper can control the contact force and torque at the end of the manipulator when executing typical tasks.
\end{abstract}

\section{Introduction}

The space manipulator can complete on-orbit assembly, observation and inspection, fault module replacement, fuel filling, and other on-orbit work. At the same time, the manipulator has the ability to adapt to microgravity, high temperature, and high radiation space environment [1]. Construction and maintenance experiences of the international space station show that the risk of astronaut extravehicular activities and work pressure is reduced, and the efficiency is improved at the same time by using space manipulators. So it is of great significance in economy and security to use space manipulator in space station [2].

Chinese space station is under construction now. As shown in Figure 1, the space station consists of CM (core module), EM (experimental module) 1, and EM 2. The CM is responsible for controlling and managing the whole space station, and EMs are mainly used in space science experiments and applications [3, 4]. Similar to the SSRMS (Space Station Remote Manipulator System) and JEMRMS (Japanese Experiment Module Remote Manipulator System) in the interna- tional space station, the Chinese space station is also equipped with a CSSRMS (Chinese Space Station Remote Manipulator System), which includes the CM manipulator and the EM manipulator. Lengths of CM manipulator and EM manipulator are about $10 \mathrm{~m}$ and $5 \mathrm{~m}$, respectively, and maximum loads are $25000 \mathrm{~kg}$ and $3000 \mathrm{~kg}$, respectively. The $\mathrm{CM}$ manipulator and the EM manipulator can work independently or work together to complete construction and maintenance tasks [5]. The CM manipulator is mainly used to complete the space station cabin relocation, spacecraft capture and docking assistance, astronauts EVA support, and so on. The EM manipulator's main tasks include exposure payload care and transport and astronauts EVA assistance [4]. In addition, one manipulator cannot cover all operating spaces, so some tasks should be completed by two manipulators' collaborative operation. For example, when installing the EM's solar wing, first, the CM manipulator takes out the solar wing from the cargo ship; then, the solar wing is transferred to the EM manipulator by the CM manipulator; at last, the EM manipulator delivers the solar wing to the rear section of experimental module to install. 




FIGURE 1: Sketch map of Chinese space station.

In some tasks of CSSRMS, such as ORU replacement, exposed payload mounting, and dual arm collaborative transport, there is a force closure due to contact between the manipulator and the operating object, and the contact force should be controlled to ensure the safety of space station and the manipulator. Force control methods of manipulators can be divided into passive compliance and active compliance. The passive compliance depends on the structural characteristics of the manipulator or the additional compliant device, while the active compliance is achieved by designing corresponding control algorithms. Passive compliance device has high specificity and poor adaptability, so its range of application is limited. There are many strategies for active compliance control, such as impedance control, force/position hybrid control, adaptive control, and intelligent control. The compliant selection matrix in the force/position hybrid control depends on environment and specific task, so the robustness of the control algorithm is poor [6]. Since the theory of adaptive control is complex, it needs more parameters at the same time, so it is rarely used in engineering. Intelligent control strategy is a new method of robot control, which uses neural network control or fuzzy control. This method is not very mature, and it has some limitations. To solve the above problems, Hogan proposed the impedance control method. The impedance control method does not control the desired force and position of the manipulator directly, but by adjusting the dynamic relationship between the end pose and the contact force. It provides a unified framework for manipulator control in free space and in compliant motion with environmental contact [7]. In 2006, Yoshida and Nakanishi introduced the impedance control in free-flying space robotics [8]. Stolfi et al. pointed out that impedance control technique presents significant advantages in grasping and capturing operations in space, and its smooth, soft operation effects have a significant advantage in limiting the perturbations on the attitude of the spacecraft [9].

The FMA (Force/Moment Accommodation Control) method is adopted to control contact force/torque for the SPDM (Special Purpose Dexterous Manipulator) on the international space station and the manipulator on the Orbital Express satellite $[10,11]$. In this method, the manipulator's control command is the end velocity in Cartesian space. A force feedback transformation matrix is designed according to the expected end impedance characteristics of the manipulator when performing contact tasks, and the contact force/torque is measured by a six axis force/torque sensor; then, the end velocity adjustment value is obtained by measured contact force/torque and force feedback transformation matrix. The FMA method is a kind of impedance control. The fine operation manipulator in JEMRMS is also equipped with a six axis wrist force/torque sensor, but the contact force is transformed into position adjustment value through selected equivalent weight, damp, and stiffness matrix, which is different with the FMA method [12]. Some researchers have made some improvements to the space manipulator impedance control algorithm. Jiang et al. propose a MHIC (Modified Hybrid Impedance Control) strategy for ORU replacements based on a ground test bed. Experimental results demonstrate that MHIC can reduce the contact force with uncertainties of constrained environments [13]. Mo et al. propose an impedance control with force signal compensation on space manipulator-assisted docking mission. Simulation experiment results indicate that this algorithm can both dock successfully and reduce the contact force effectively [14].

The rest of this paper is organized as follows. In Section 2, task flows of typical tasks that require force compliance control is analyzed, including ORU payload changeout and dual arm coordinated payload transport; Section 3 designs the force compliance control algorithm in detail to perform these tasks; in Section 4, the ground experimental platform to verify the force compliance control method is built using two industrial manipulators; Section 5 presents the experimental results of typical tasks, and results are discussed; conclusions are given in the last section.

\section{Typical On-Orbit Missions Analysis}

This paper mainly studies the force compliance control method of ORU payload changeout and dual arm collaborative payload transport; therefore, the task flow of the above two tasks is analyzed in detail firstly.

2.1. ORU Payload Changeout. In Chinese space station, the ORU is generally mounted on the passive part of a PCM (payload connecting mechanism). On one side of the passive part of a PCM, there is an EM manipulator end effector 


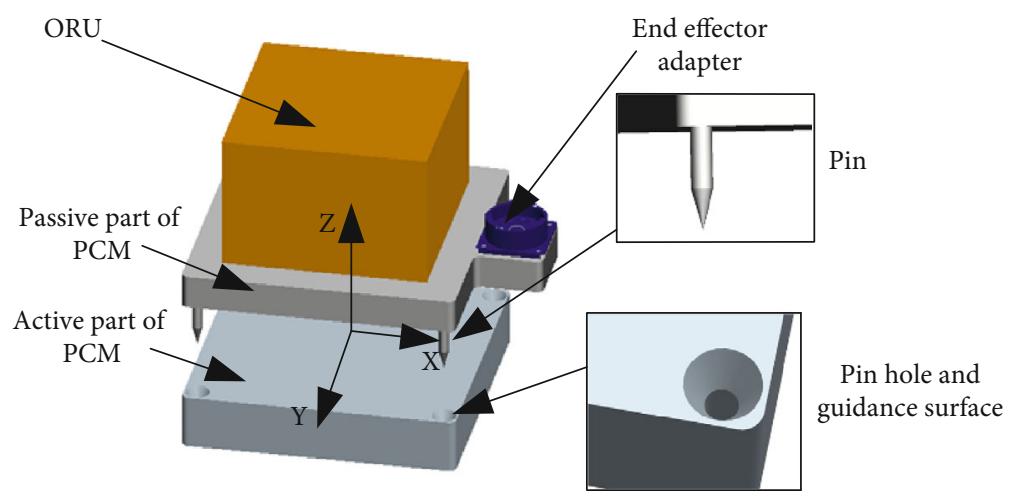

FIGURE 2: ORU installation sketch map.



(a)

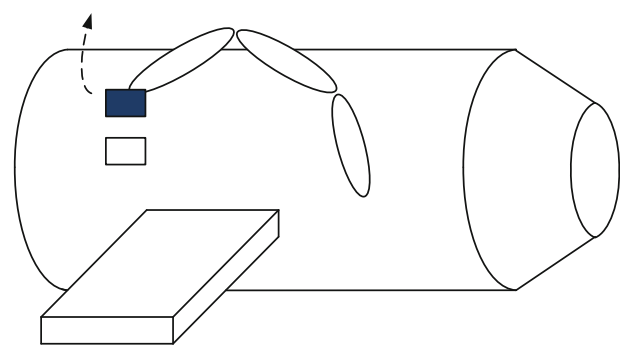

(c)

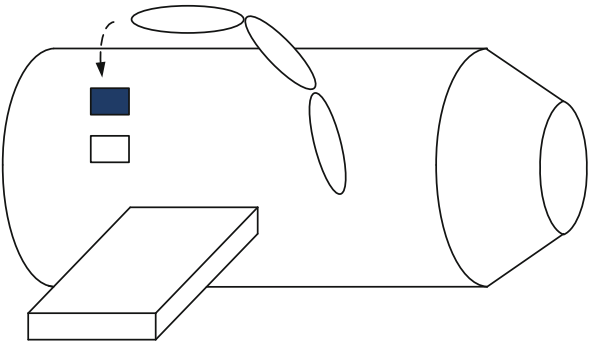

(b)

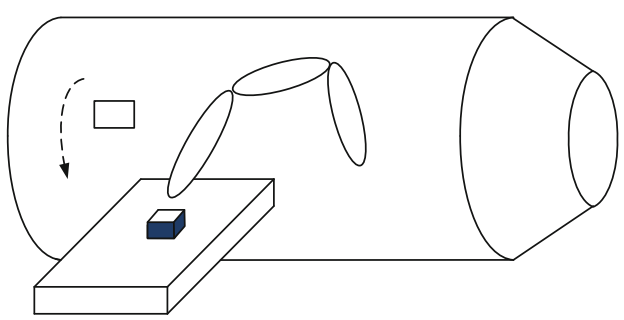

(d)

FIGURE 3: ORU changeout task flows: (a) old ORU extracting; (b) old ORU inserting; (c) new ORU extracting; (d) new ORU installing.

adapter or a CM manipulator end effector adapter, and on the other side, there are some guiding pins (Figure 2). The active part of the PCM is fixed on the exposed platform, and there are some pinholes with guide surfaces corresponding to pins on the passive part, and there is a locking mechanism to lock the passive part. One of the available schemes for the EM manipulator end effector is similar to that of the ERA (European Robotic Arm) end effector, which has an inclined guide surface to correct the initial deviation between the manipulator end effector and the adapter [15].

There are 4 stages in an ORU changeout task flow, which are old ORU extracting, old ORU inserting, new ORU extracting, and new ORU installing (Figure 3). In the old ORU extracting stage, the space station manipulator grasps and pulls out an old ORU from the active part of PCM installed on the exposed platform. In the second stage, the space station manipulator with the captured old ORU moves from the initial pose, which is the end pose of the first stage, to the top of the active part of PCM which is in the air-lock cabin, and inserts the old ORU into the PCM. In the third stage, a new ORU is extracted from another PCM in the air-lock cabin. In the last stage, the manipulator with the captured new ORU moves from the air-lock cabin to the exposed platform, and the new ORU is inserted into the PCM on the exposed platform [16].

Every stage can be divided into 4 substages, which are free motion stage, approximate motion stage, docking/releasing motion stage, and inserting/pulling out motion stage [15]. In free motion stage, the manipulator just needs to move from an initial pose to the vicinity of the ORU to be replaced or the active part of PCM. There is a safe distance between the manipulator end effector and the surrounding environment, and the manipulator uses position or speed control to achieve desired pose.

In approximate motion stage, the manipulator gets close to the target under the guidance of a visual camera. At the end of the approximate motion stage, the end effector must be in the adapter's guidance scope, or the pin on the passive part of the PCM must be in the pinhole's guidance scope. In approximate motion stage, an accidental contact may occur because of motion error, environment changes, or other factors. Therefore, the manipulator's end should be compliant in every direction to avoid large contact force between the manipulator and the environment. 


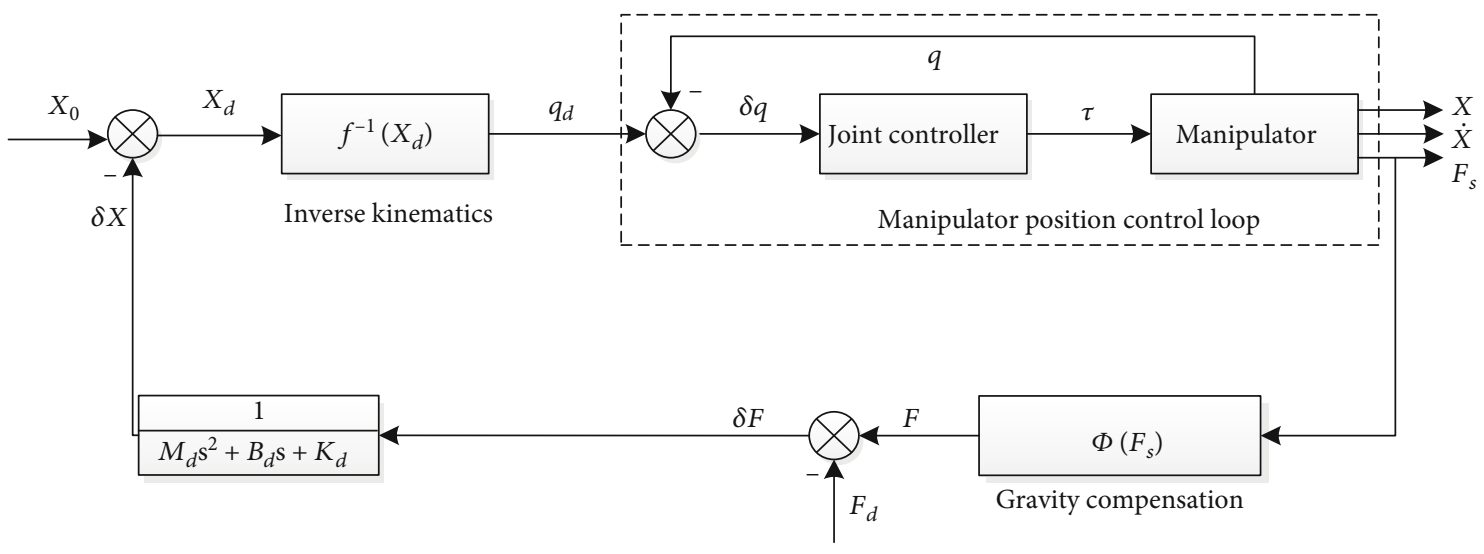

FIgURE 4: Schematic diagram of position-based impedance control.

In the docking motion stage, the manipulator end effector further approaches the adapter along the adapter normal direction and the guidance surface of the end effector contacts with the adapter firstly. Under the influence of contact force, the initial deviation between the effector and the adapter is corrected continuously by the guiding surface, and the effector locks the adapter until the effector is fully inserted. In the normal direction, the manipulator is required to have a greater stiffness to ensure that the effector is continuously close to the adapter. In other directions, there are motion constraints between the end effector and the adapter, so the manipulator should be compliant. In releasing stage, the manipulator uses position control along the normal direction of the adapter, and through motion planning, the manipulator carries the end-effector away from the adapter in a straight line along the normal direction of the adapter. At the same time, in order to ensure the safety of release operation, the manipulator adopts force compliance control strategy to control the contact force/torque in other directions.

The inserting motion stage refers to the process that the manipulator inserts the passive part of the PCM with an ORU into the PCM active part, and eventually, the ORU is fixed on the active part. Similar to the docking motion stage, pins on the passive part contact with pinholes' guiding surface on the active part firstly if there is pose error. Under the influence of contact force, the initial deviation of pins and pinholes is corrected continuously by the guiding surface, until all pins are fully inserted into the pinhole. At last, the active part locks the passive part completely. To ensure that the passive part is continuously close to the active part, the manipulator is required to have a greater stiffness in the normal direction of the PCM active part. In other direction, there are motion constraints between the end of the pin and the pinhole, so the manipulator should be compliant. The pulling out motion stage is the opposite.

2.2. Dual Arm Collaborative Payload Transport. In the construction and maintenance process of the Chinese space station, large payloads carried by the cargo spacecraft often should be transported to the designated location of the space station, but the CM manipulator's operation space cannot cover the entire space station, so these tasks should be finished by two manipulators' cooperation. Taking the EM solar wing transportation as an example, the $\mathrm{CM}$ manipulator first takes out the solar wing from the cargo ship docked with the $\mathrm{CM}$ and delivers it to the handover position, where the EM manipulator also can reach, and then, each joint of the $\mathrm{CM}$ manipulator brakes. After that, the CM manipulator waits for the EM manipulator to fetch the solar wing. Similar to the ORU changeout process in Section 2.1, the flow of EM manipulator getting the solar wing can be divided into free motion stage, approximate motion stage, docking motion stage, two arm close chain motion stage, and pulling out motion stage.

In the free motion stage, the EM manipulator gets close to the solar wing at the end of the CM manipulator. Since the actual length of the CM manipulator reaches $10 \mathrm{~m}$, it has a strong flexibility, and it vibrates even in the case of braking. In the approximate motion stage, the EM manipulator tracks and further approaches the EM manipulator adapter on the solar wing under the guidance of a visual camera. At the end of this stage, the EM manipulator adapter on the solar wing enters into the EM manipulator effector's capture range. The EM manipulator should be compliant in all directions, so as to avoid greater contact force between the robot arm and the environment to ensure safety. During the docking stage, the EM manipulator end effector captures and locks the EM manipulator adapter on the solar wing. In the adapter's normal direction, the manipulator is required to have a greater stiffness. In other direction, the manipulator should be compliant. After the docking stage, the CSSRMS enters into the close chain motion mode. Due to the vibration of the CM manipulator, the EM manipulator shall be in compliant control mode in all directions, and the EM manipulator follows the CM manipulator passively. The pulling out motion stage refers to the process, in which the EM manipulator pulls the CM manipulator adapter on the solar wing from the CM manipulator end effector. In this stage, the EM manipulator control requirements are similar to the docking stage, but the motion direction is the opposite.

\section{Compliance Control Method Research}

3.1. Compliance Control Algorithm Design. On the basis of the above analysis, this paper adopts a position-based impedance control method, and the control principle block diagram is shown below (Figure 4). In Figure $4, X_{0}$ is the 
Cartesian space target pose command of the space station manipulator; $f^{-1}$ is the inverse kinematics of the manipulator; $F$ is the pure contact force between the end of the manipulator and the environment, and $F_{d}$ is the expected contact force. $M_{d} s^{2}+B_{d} s+K_{d}$ is the expected impedance characteristic of the system. $M_{d}, B_{d}$ and $K_{d}$ are the expected inertia matrix, damping matrix, and stiffness matrix. $M_{d}, B_{d}$ and $K_{d}$ are all $N$ order diagonal matrices, and $N$ represents the freedom of the manipulator.

As can be seen from the Figure 4, because of the difference $\delta \boldsymbol{F}$ between the actual contact force and the expected contact force, the pose adjustment value $\delta \boldsymbol{X}$ in the Cartesian space of the manipulator is

$$
\delta \boldsymbol{X}=\frac{\delta \boldsymbol{F}}{\boldsymbol{M}_{d} s^{2}+\boldsymbol{B}_{d} s+\boldsymbol{K}_{d}} .
$$

According to Cartesian space pose command $X_{0}$ and the pose adjustment value $\delta \boldsymbol{X}$, the desired pose of the manipulator $X_{d}$ is obtained. Then, joint commands are obtained by the inverse kinematics and sent to joints' controllers.

Through detailed analysis of on-orbit tasks in Section 2, in approximate motion stage and close chain motion stage, the manipulator's end should be compliant in every direction, so the Cartesian space pose adjustment value $\delta \boldsymbol{X}$ is

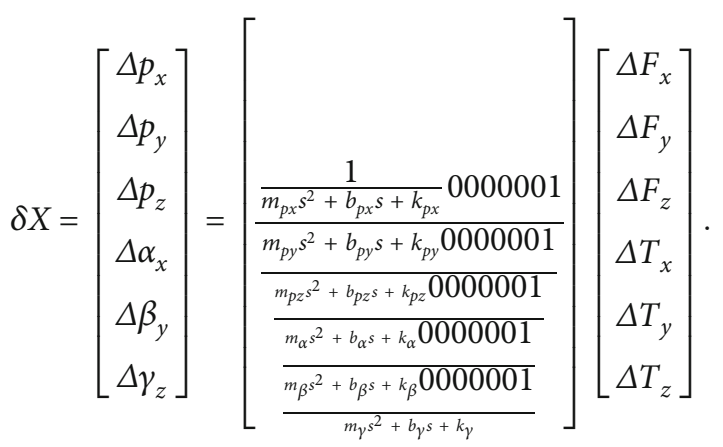

In equation (2), $\Delta P_{x}, \Delta P_{y}$, and $\Delta P_{z}$ are position adjustment value along the $x, y$, and $z$ directions in Cartesian space. $\Delta \alpha_{x}$, $\Delta \beta_{y}$, and $\Delta \gamma_{z}$ are adjustment value of attitude angles. $\Delta F_{x}$, $\Delta F_{y}$, and $\Delta F_{z}$ are contact force along the $x, y$, and $z$ directions. $\Delta T_{x}, \Delta T_{y}$, and $\Delta T_{z}$ are contact torque. $m_{p x}, m_{p y}, m_{p z}, m_{p \alpha}, m_{p \beta}$, and $m_{p \gamma}$ are expected inertia parameter by impedance control in all directions along the six-dimensional force/torque sensor coordinate system. $b_{p x}, b_{p y}, b_{p z}, b_{p \alpha}, b_{p \beta}$, and $b_{p \gamma}$ are expected damping parameter by impedance control in all directions along the six-dimensional force/torque sensor coordinate system. $k_{p x}, k_{p y}, k_{p z}, k_{p \alpha}, k_{p \beta}$, and $k_{p \gamma}$ are expected stiffness parameter by impedance control in all directions along the six-dimensional force/torque sensor coordinate system.

In docking/releasing and inserting/pulling out motion stage, to ensure the effector approaching or getting away from the adapter along the normal direction of the adapter, or the passive part of the PCM approaching or getting away from the active part along the normal direction of the active part, the position control mode is used in this direction to get a high stiffness and accuracy. At the same time, the contact force should be monitored in the normal direction. When the contact force is found to be too large, the motion will be stopped. In other directions, there are motion constraints, so the impedance control method above is used to prevent a high contact force/torque. If the normal direction of the adapter and the active part of the PCM is $Z$ axis, then,

$$
\delta X=\left[\begin{array}{c}
\Delta p_{x} \\
\Delta p_{y} \\
\Delta p_{z} \\
\Delta \alpha_{x} \\
\Delta \beta_{y} \\
\Delta \gamma_{z}
\end{array}\right]=\left[\frac{\frac{1}{m_{p x} s^{2}+b_{p x} s+k_{p x}} 0000001}{\frac{m_{p y} s^{2}+b_{p y} s+k_{p y} 0000010000001}{\frac{m_{\alpha} s^{2}+b_{\alpha} s+k_{x} 0000001}{m_{\beta^{2}}+b_{\beta} s+k_{\beta} 000001}}}\right]\left[\begin{array}{c}
\Delta F_{x} \\
\Delta F_{y} \\
\Delta p_{z} \\
\Delta T_{x} \\
\Delta T_{y} \\
\Delta T_{z}
\end{array}\right] .
$$

In one control cycle, the position adjustment value $\Delta P_{z}$ in the $Z$ direction is $\Delta$ step, which is given in advance according to the task execution time. When the contact force along the $Z$ direction is greater than a given threshold value $F_{t z}$ and the limit switch is triggered at the same time, it can be decided that the docking or the inserting motion stage finishes, so the manipulator stops. It must be pointed out that equation (3) is only applicable to plug and extract operations along the $Z$ direction and inevitably has certain limitations. However, under the known operating object and environment, such as the ORU replacement operation of the space station, this method can be used to complete the operation task.

3.2. Discretization of the Impedance Control Algorithm. In order to implement in a computer controller, the impedance control algorithm in Section 3.1 needs to be discretized. According to equation (1),

$$
\delta F=M_{d} \delta \ddot{X}+B_{d} \delta \dot{X}+K_{d} \delta X .
$$

$\delta \dot{\boldsymbol{X}}$ and $\delta \ddot{\boldsymbol{X}}$ in equation (4) can be expressed as,

$$
\begin{aligned}
& \delta \dot{\boldsymbol{X}}(\mathrm{k})=\frac{\delta \boldsymbol{X}(\mathrm{k})-\delta \boldsymbol{X}(\mathrm{k}-1)}{\mathrm{T}}, \\
& \frac{\delta \ddot{\boldsymbol{X}}(\mathrm{k})=\delta \boldsymbol{X}(\mathrm{k})+\delta \boldsymbol{X}(\mathrm{k}-2)-2 \delta \boldsymbol{X}(\mathrm{k}-1)}{\mathrm{T}^{2}} .
\end{aligned}
$$

In equations (5) and (6), $T$ is the system sampling period. $\delta \boldsymbol{X}(\mathrm{k}), \delta \boldsymbol{X}(\mathrm{k}-1)$, and $\delta \boldsymbol{X}(\mathrm{k}-2)$ are pose adjustment value in the current sampling cycle and last two sampling cycles. $\delta \boldsymbol{F}$ (k) can expressed as equation (7) by substituting the equations (5) and (6) into equation (4),

$$
\frac{\frac{\delta \boldsymbol{F}(\mathrm{k})=\boldsymbol{M}_{d}+\boldsymbol{B}_{d} \mathrm{~T}+\boldsymbol{K}_{d} \mathrm{~T}^{2}}{\mathrm{~T}^{2} \delta \boldsymbol{X}(\mathrm{k})-2 \boldsymbol{M}_{d}+\boldsymbol{B}_{d} \mathrm{~T}}}{\mathrm{~T}^{2} \delta \boldsymbol{X}(\mathrm{k}-1)+\frac{\boldsymbol{M}_{d}}{\mathrm{~T}^{2}} \delta \boldsymbol{X}(\mathrm{k}-2) .}
$$

Let us take the $x$ direction as an example (the discretization 
in the other direction is the same as in the $x$ direction), the pose adjustment value in the current sampling cycle $\delta \boldsymbol{X}_{x}(\mathrm{k})$ is

$$
\delta \boldsymbol{X}_{x}(\mathrm{k})=\boldsymbol{a}_{0} x \delta \boldsymbol{F}_{x}(\mathrm{k})+\boldsymbol{a}_{1} x \delta \boldsymbol{X}_{x}(\mathrm{k}-1)+\boldsymbol{a}_{2} x \delta \boldsymbol{X}_{x}(\mathrm{k}-2)
$$

In equation (8), $\delta \boldsymbol{F}_{x}(\mathrm{k}), \delta \boldsymbol{X}_{x}(\mathrm{k}-1)$, and $\delta \boldsymbol{X}_{x}(\mathrm{k}-2)$ are components in the $x$ direction of $\delta \boldsymbol{F}(\mathrm{k}), \delta \boldsymbol{X}(\mathrm{k}-1)$, and $\delta \boldsymbol{X}$ $(\mathrm{k}-2)$. Equation (8) shows that the current pose adjustment value $\delta \boldsymbol{X}_{\boldsymbol{x}}(k)$ has a relationship with current force deviation $\delta \boldsymbol{F}_{\boldsymbol{x}}(k)$ and the last two sampling cycle's pose adjustment value $\delta \boldsymbol{X}_{x}(k-1)$ and $\delta \boldsymbol{X}_{x}(k-2)$. When calculating the first and second pose adjustment value, it can be assumed that $\delta \boldsymbol{X}_{\boldsymbol{x}}(-1)$ and $\delta \boldsymbol{X}_{\boldsymbol{x}}(-2)$ are zero.

$$
\frac{\boldsymbol{a}_{0} x=\mathrm{T}^{2}}{\frac{m_{p x}+b_{p x} \mathrm{~T}+\mathrm{k}_{p x} \mathrm{~T}^{2}, \boldsymbol{a}_{1} x=2 m_{p x}+b_{p x} \mathrm{~T}}{m_{p x}+b_{p x} \mathrm{~T}+\mathrm{k}_{p x} \mathrm{~T}^{2}, \boldsymbol{a}_{2} x=\frac{-m_{p x}}{m_{p x}+b_{p x} \mathrm{~T}+\mathrm{k}_{p x} \mathrm{~T}^{2}}}} .
$$

3.3. Selection of Impedance Control Parameters. In order to determine the reasonable impedance parameters $M_{d}, B_{d}$, and $K_{d}$, the one-dimensional impedance control model is analyzed on the basis of equation (4), and the simplified two order system model is expressed as:

$$
m_{d} \ddot{e}+b_{d} \dot{e}+k e=f \text {. }
$$

$\ddot{e}, \dot{e}$, and $e$ are acceleration, velocity, and position adjustment value in single-dimensional space. $f$ is the pure disturbance force. $m_{d}, b_{d}$, and $k_{d}$ are the expected inertia, damping, and stiffness. The following transfer function is obtained by doing a Laplace transformation on equation (10).

$$
\frac{\frac{e(s)}{f(s)}=\mu \omega_{0}{ }^{2}}{s^{2}+2 \xi \omega_{0} s+\omega_{0}^{2}} .
$$

In equation (11),

$$
\begin{aligned}
& \frac{\mu=1}{k_{d}}, \\
& \xi=\frac{b_{d}}{2 \sqrt{m_{d} k_{d}}}, \\
& \omega_{n}=\sqrt{\frac{k_{d}}{m_{d}}} .
\end{aligned}
$$

$\xi$ is the damping ratio, and $\omega_{n}$ is the natural frequency of the impedance control system. $\xi$ plays an important role on the control system stability and overshoot of the contact force. The increase of $\xi$ can improve the stability of the control system, but increase contact force overshoot at the same time. In practice, the target stiffness $k_{d}$ of the impedance control is determined firstly according to the steady-state pose error and the maximum allowable contact force error. If the environment stiffness is $k_{E}$, the target stiffness $k_{d}$ should be far less than the environment stiffness, and the damping ratio $\xi$ needs to satisfy the following equation (15) [17],

$$
\frac{\xi \geq \sqrt{1}+k_{E} / k_{d}-1}{2 .}
$$

According to experiences, the damping ratio $\xi$ is generally between 1 and 15 [18], and then, $\omega_{n}$ is determined according to operation task demand and system signal delay. At last, the desired inertia $m_{d}$ and damping $b_{d}$ are determined according to equations (13) and (14).

3.4. Real-Time Gravity Compensation. Because the six dimension force/torque sensor is installed between the manipulator end joint and the end effector, so if the compliance control experiment is done on the ground, the force/torque sensor measurement value $F_{s}$ not only includes the pure contact force $F$ and the gravity of the end effector but also contains payload's gravity when the payload is captured. Because the impedance control algorithm's input is the pure contact force $F$, the gravity terms must be compensated. If the force compliance control is in the weightless environment of space, the gravity does not need to be compensated. The homogeneous transformation matrix between the force/torque sensor coordinate and the base coordinate of the manipulator is



In equation (16), ${ }^{\text {end }} T_{\text {sensor }}$ is the homogeneous transformation matrix between the force/torque sensor coordinate and the end effector coordinate, which is known before by assembly relationship. ${ }^{\text {base }} T_{\text {end }}$ can be calculated by forward kinematics. If the gravity field in the base coordinate system is $[0,0,-g]^{T}$, the pure contact force vector $F$ is,

$$
F=F_{s}-\left[\begin{array}{c}
R_{f} \cdot[0,0,-m g]^{T} \\
\vec{r}_{c} \times R_{f} \cdot[0,0,-m g]^{T}
\end{array}\right] .
$$

In equation (17), when there is not a payload at the end, $\vec{r}_{c}$ is the vector of the centroid of the end effector relative to the origin of the force/torque sensor coordinate. When there is a payload at the manipulator's end, $\vec{r}_{c}$ is the vector of the centroid of the assembly composed by the end effector and the payload relative to the origin of the force/torque sensor coordinate.

\section{Ground Experimental Verification Platform}

A ground experiment verification platform is designed to validate the design of the compliance control algorithm. The platform includes force/torque sensor acquisition system, simulated EM manipulator, simulated CM manipulator, simulated end effector, simulated ORU, and simulated exposed platform. Among them, the simulated EM manipulator's 


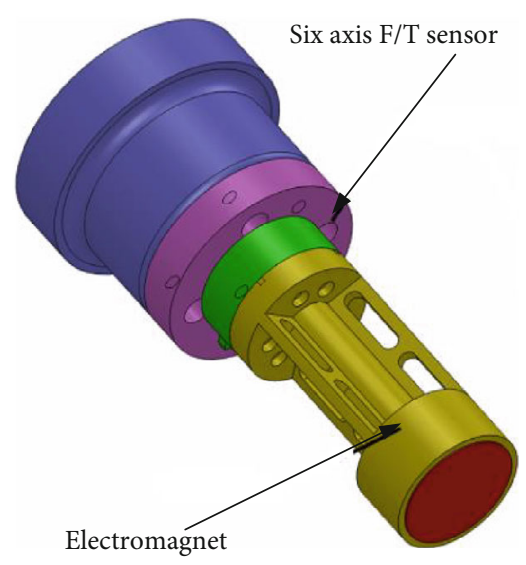

(a)

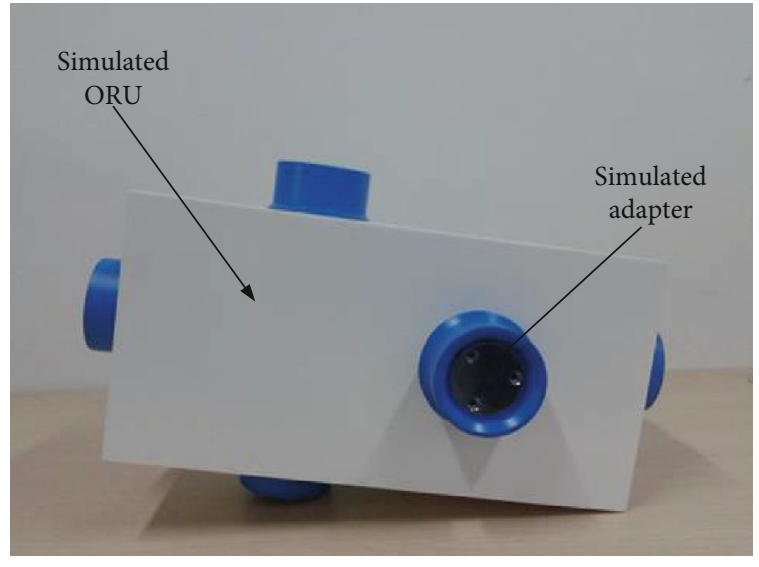

(b)

FIGURE 5: Simulated end effector, ORU, and adapter: (a) simulated end effector; (b) simulated ORU and adapter.

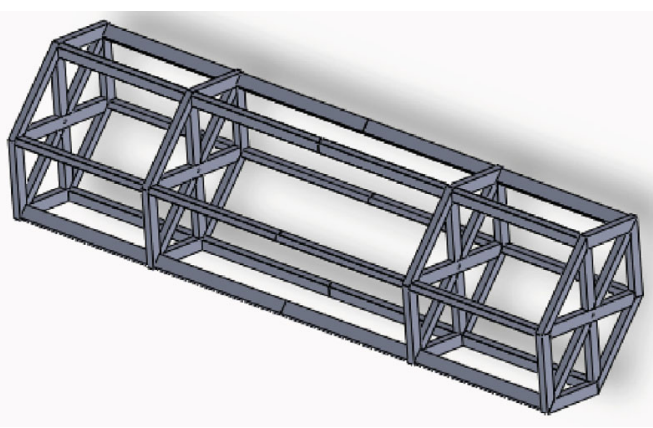

(a)

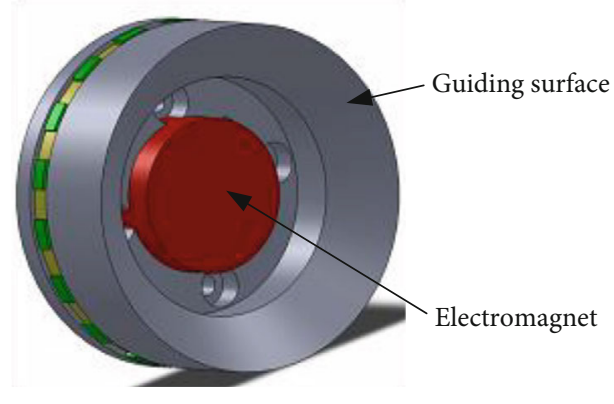

(b)

FIGURE 6: Simulated exposed platform and payload connecting mechanism: (a) simulated exposed platform; (b) simulated active part of PCM.

length is $0.9 \mathrm{~m}$, and its rated load is $5 \mathrm{~kg}$. Simulated CM manipulator's length is $1.4 \mathrm{~m}$, and its rated load is $8 \mathrm{~kg}$.

A simulated end effector is mounted at the end of the simulated EM manipulator (Figure 5(a)). Because the interface between the end effector and the payload of the EM manipulator is circular, the simulated end effector is also designed to be circular. The simulated end effector's locking mechanism is realized by an electromagnet and its largest suction force is $250 \mathrm{~N}$, which is controlled by the I/O port of the central controller of the simulated EM manipulator. A six axis force/torque sensor whose model is Mini45 SI290-10 is mounted between the end effector and the last joint of the simulated EM manipulator. The sensor's force measurement range is $290 \mathrm{~N}$, and the torque measurement range is $10 \mathrm{Nm}$. The sensor's output signal is adjusted by a signal amplifier, and then, the data is collected by NI 6220 and sent to the simulated EM manipulator's central controller.

According to the scaling ratio between the real space station manipulator and the simulated EM manipulator, the simulated ORU payload is designed as a cuboid of $300 \mathrm{~mm}$ $* 150 \mathrm{~mm} * 150 \mathrm{~mm}$, and simulated adapters are installed on 6 sides of the ORU payload (Figure 5(b)). According to the interface shape and principle of the EM manipulator end effector, the simulated adapter is also designed to be cylindrical, with an outer diameter of $50 \mathrm{~mm}$ and an inner diameter of $30 \mathrm{~mm}$. The simulated end effector can insert into and lock the adapter by an electromagnet. A $10 \mathrm{~mm}$ chamfer is designed at the opening of the simulated adapter to simulate the guiding surface of the real EM manipulator end effector.

A simple exposed platform is constructed with truss structure in the platform (Figure 6(a)). Because the real PCM adopts the circular pinhole with a guide surface to match the pin, the design of the active part of the simulated PCM as shown in Figure 6(b) is also designed to be circular. A guiding surface is designed at the opening of the active part of the simulated PCM. Its diameter is $80 \mathrm{~mm}$, and the chamfer of the guiding surface is $30 \mathrm{~mm}$. The passive part of the simulated PCM is the same with the adapter in Figure 5(b).

\section{Experiment Results and Discussion}

This section first verifies the correctness of the gravity compensation algorithm and force compliance control algorithm; then, ORU replacement and dual arm collaborative payload transport experiments are carried out on the platform.

5.1. The Force Compliance Control Algorithm Verification. To verify the force compliance control algorithm, the end effector pose is set to a fixed value, and the manipulator is set in force compliance control mode. An external force is applied to the end of the manipulator. The force/torque in each direction 

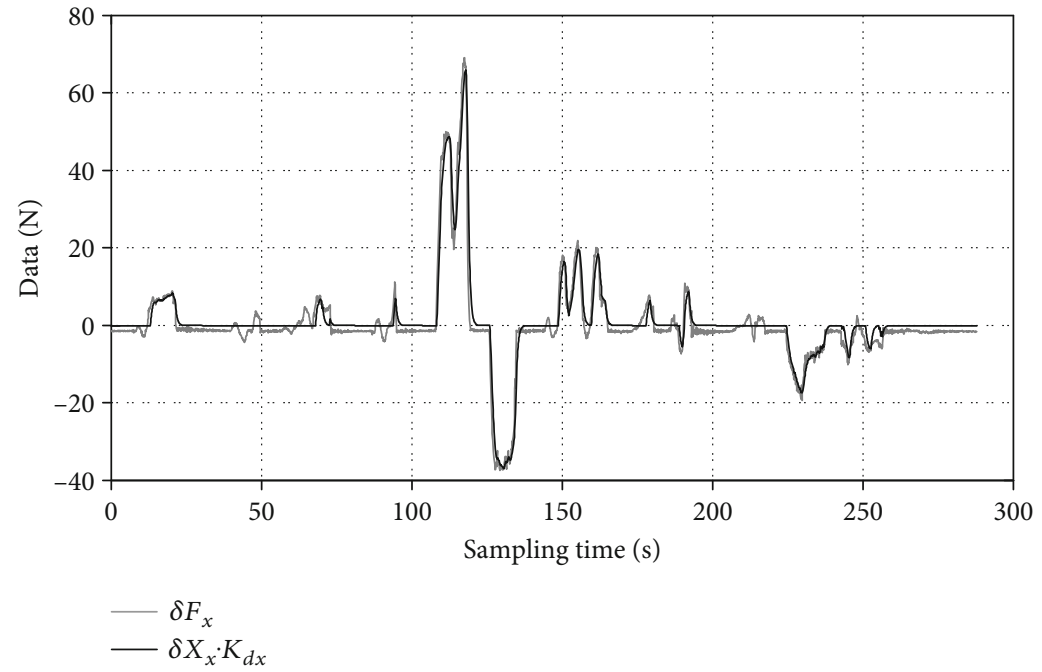

(a)

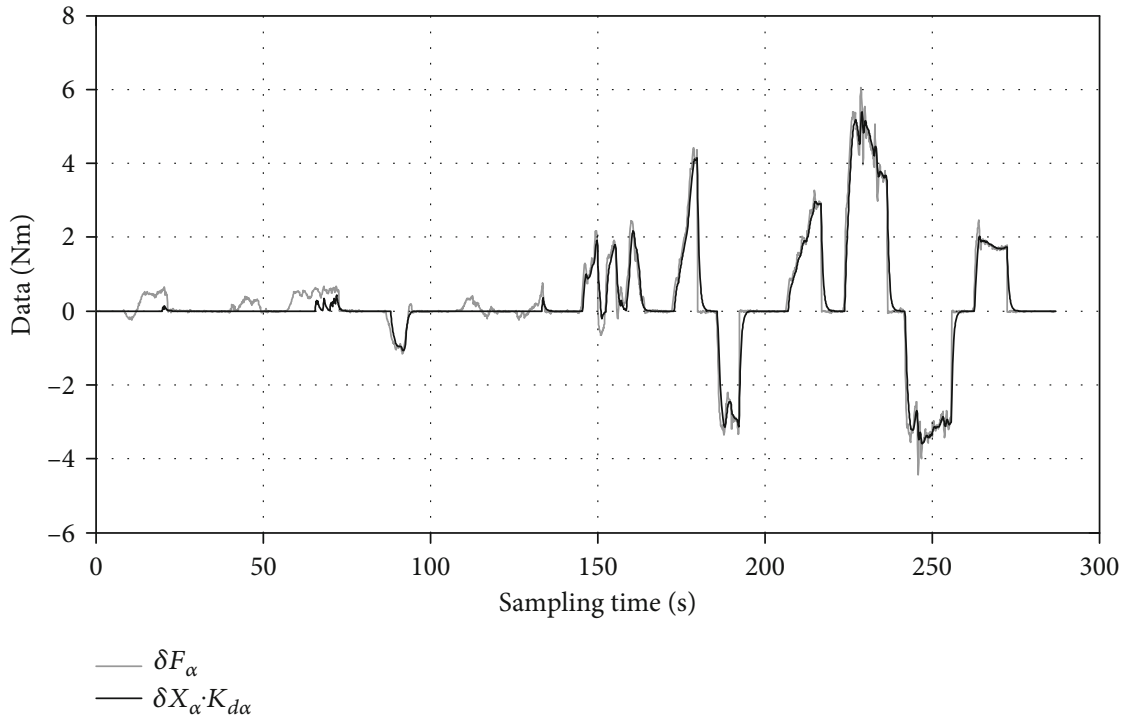

(b)

Figure 7: Continued. 


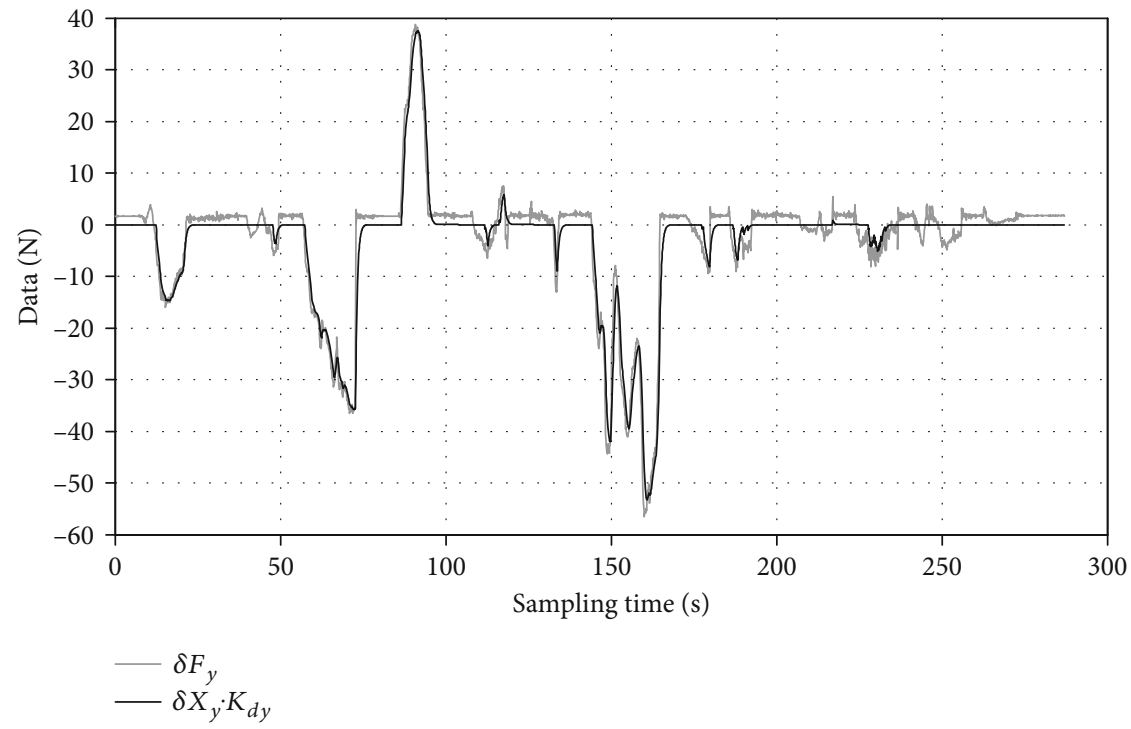

(c)

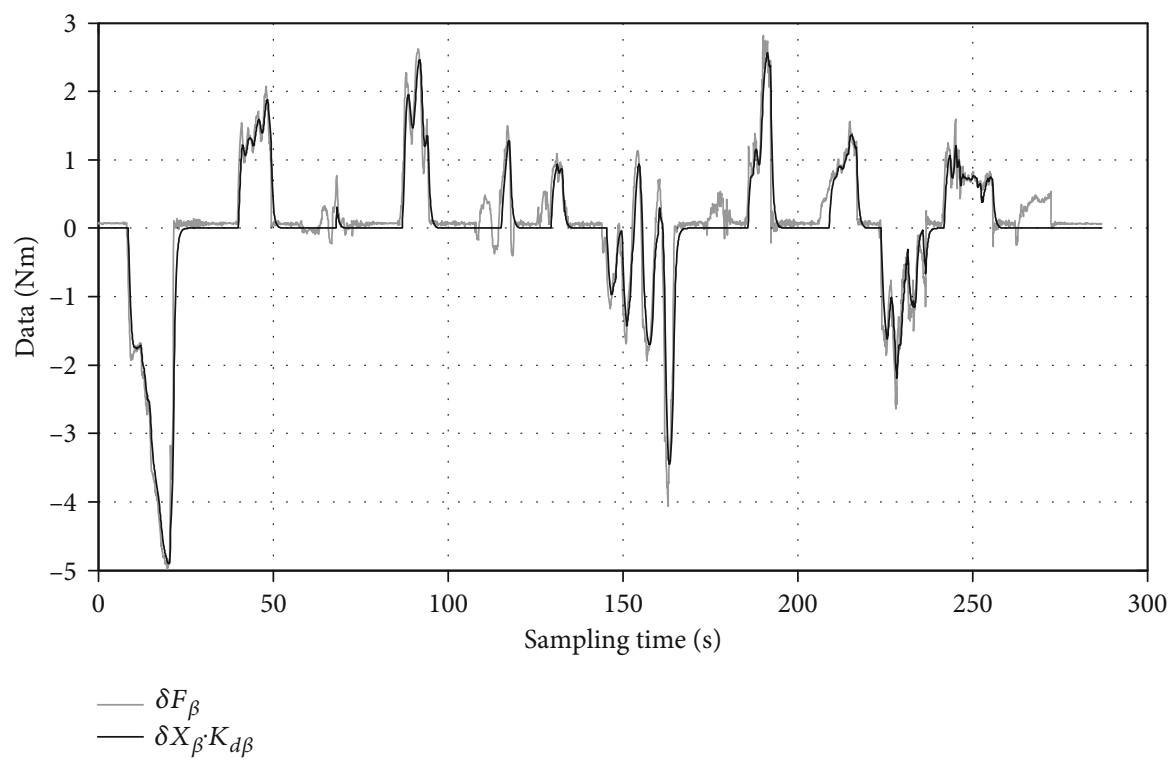

(d)

FIgURE 7: Continued. 




$-\delta F_{z}-\delta X_{z} \cdot K_{d z}$

(e)

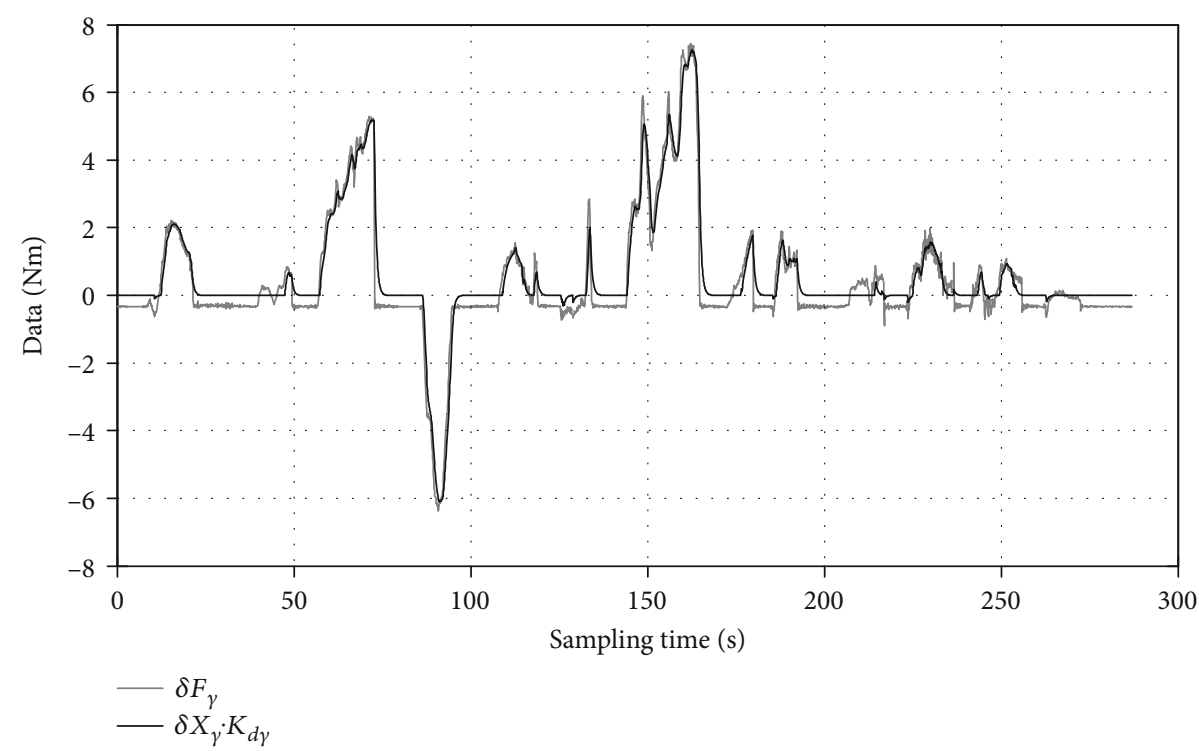

(f)

FIGURE 7: Force compliance control experiments results: (a) force in $X$ direction; (b) torque around $X$ axis; (c) force in $Y$ direction; (d) torque around $Y$ axis; (e) force in $\mathrm{Z}$ direction; (f) torque around $Z$ axis.

of the manipulator after gravity compensation and the corresponding pose adjustment value are recorded. In this experiment, the impedance parameters are determined as:



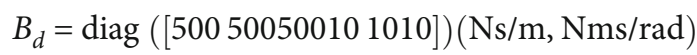

$$
\begin{aligned}
& K_{d}=\operatorname{diag}([100010001000202020])(\mathrm{N} / \mathrm{m}, \mathrm{Nm} / \mathrm{rad})
\end{aligned}
$$

The expected contact force and torque of the manipulator

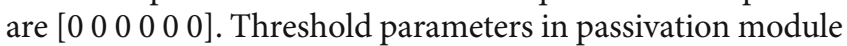
are $4 \mathrm{~N}\left(F_{N}\right)$ and $0.5 \mathrm{Nm}\left(T_{N}\right)$. There are two curves in

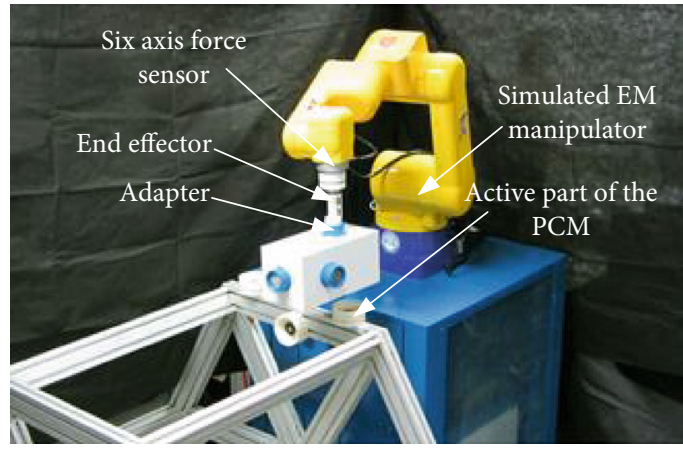

FIGURE 8: ORU payload replacement experiment. 


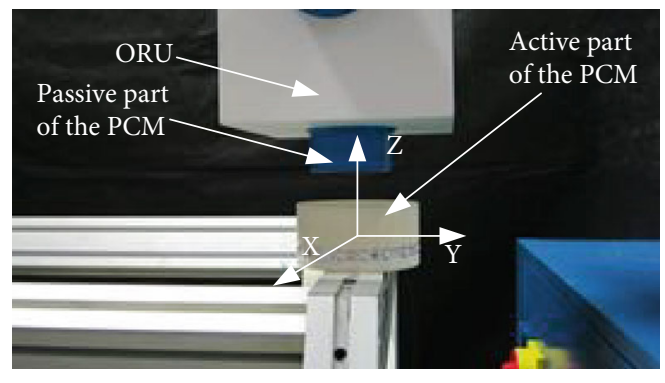

(a)



(c)

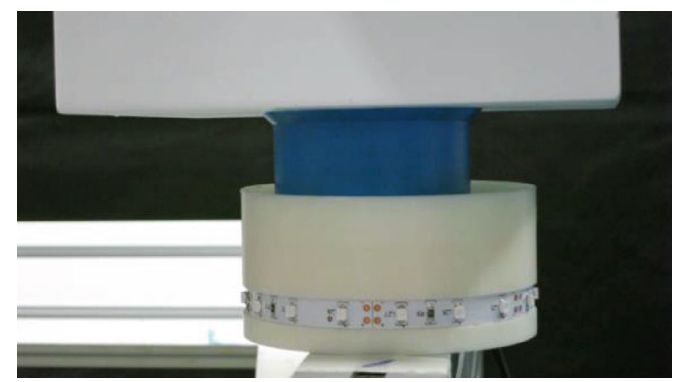

(b)

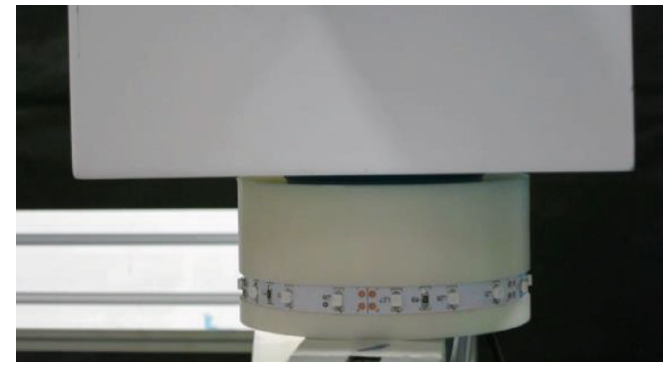

(d)

FIGURE 9: Inserting of the ORU: (a) initial state; (b) start to contact with active part; (c) insert into the active part absolutely; (d) the active part lock the passive part.

Figure 7. One curve's horizontal ordinate is the sampling time, and the longitudinal ordinate is the actual value of the pure contact force $\delta \boldsymbol{F}$ after gravity compensation. The other curve's horizontal ordinate is the sampling time too, but the longitudinal ordinate is the end pose adjustment value $\delta X$ multiplied by the desired stiffness $K_{d}$. From Figure 7 , it can be seen that the manipulator is stable and is fixed at the initial position in the absence of external force, and the end pose adjustment value is 0 . When subjected to external force, the impedance control algorithm makes the manipulator's end pose to follow the pure contact force rapidly and accurately, and experiment results show a good control effect. The above results verify the correctness of the force compliance control algorithm, and the choice of control parameters is reasonable.

5.2. ORU Replacement Experiment. The ORU payload replacement experiment is shown in Figure 8. Because the impedance control method is the same in stages of old ORU extracting, old ORU inserting, new ORU extracting, and new ORU installing, therefore, this section only analyzes the experimental results of ORU inserting stage.

Before the experiment, the end effector of the simulated EM manipulator with a docked ORU reached to the top of the active part of the PCM mounted on the truss (Figure 9(a)). The passive part of the PCM is in the active part's capture range, that is, when the EM manipulator moves along the normal direction of the active part of the PCM ( $Z$ direction), the passive part mounted under the ORU will contact with the guiding surface of the active part of the PCM. When the ORU inserting stage begins, the EM manipulator moves along the $\mathrm{Z}$ direction and the passive part of the PCM contacts with the guiding surface of the active part (Figure 9(b)). The pose of the EM manipulator is adjusted continuously by the contact force at the chamfer of the active part of the PCM, until the passive part is inserted into the active part absolutely (Figure 9(c)). Then, the electromagnet in the active part is energized, and the active part and the passive part of the PCM are locked, which means that the ORU inserting stage is over (Figure 9(d)).

Figure 10 is the pure contact force and torque obtained during the inserting stage. Figure 11 is the real-time end pose of the manipulator during the inserting stage. The sampling period of the force/torque sensor is $2 \mathrm{~ms}$, and the impedance control cycle is $20 \mathrm{~ms}$. In the former $8.5 \mathrm{~s}$, in directions except $Z$ axis, because the simulated EM manipulator is in a force compliance control mode, the end pose of the manipulator is adjusted to follow the changes of the pure contact force and torque, so that the contact force and torque always oscillate near 0 . Because the manipulator is in position control mode in the $Z$ direction, the position in this direction varies linearly, and the active and passive part of the PCM is kept close to each other. After $8.5 \mathrm{~s}$, the passive part of the PCM is fully inserted into the active part, and the contact force along the $Z$ direction increases rapidly until it reaches the set threshold $-2 \mathrm{~N}$, indicating that the insertion process has been completed. Then, the manipulator stops moving, and the position, the contact force, and the torque remain unchanged.

\subsection{Dual Arm Collaborative Payload Transport Experiment.} The dual arm collaborative payload transport experiment is shown in Figure 12. In the experiment platform, the left is the simulated CM manipulator, and the right is the simulated EM manipulator.

At the beginning of the experiment, the payload to be transported is captured by the simulated CM manipulator, and the simulated $\mathrm{CM}$ manipulator moves slightly to 

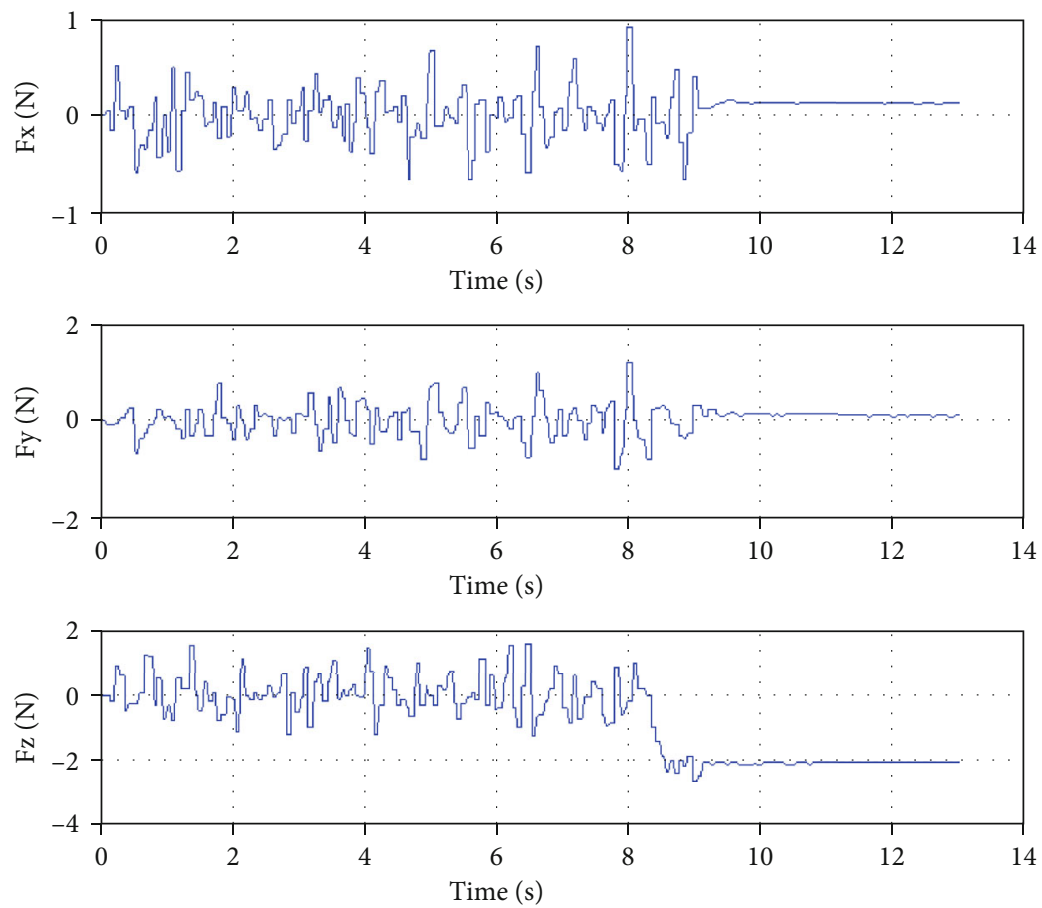

(a)
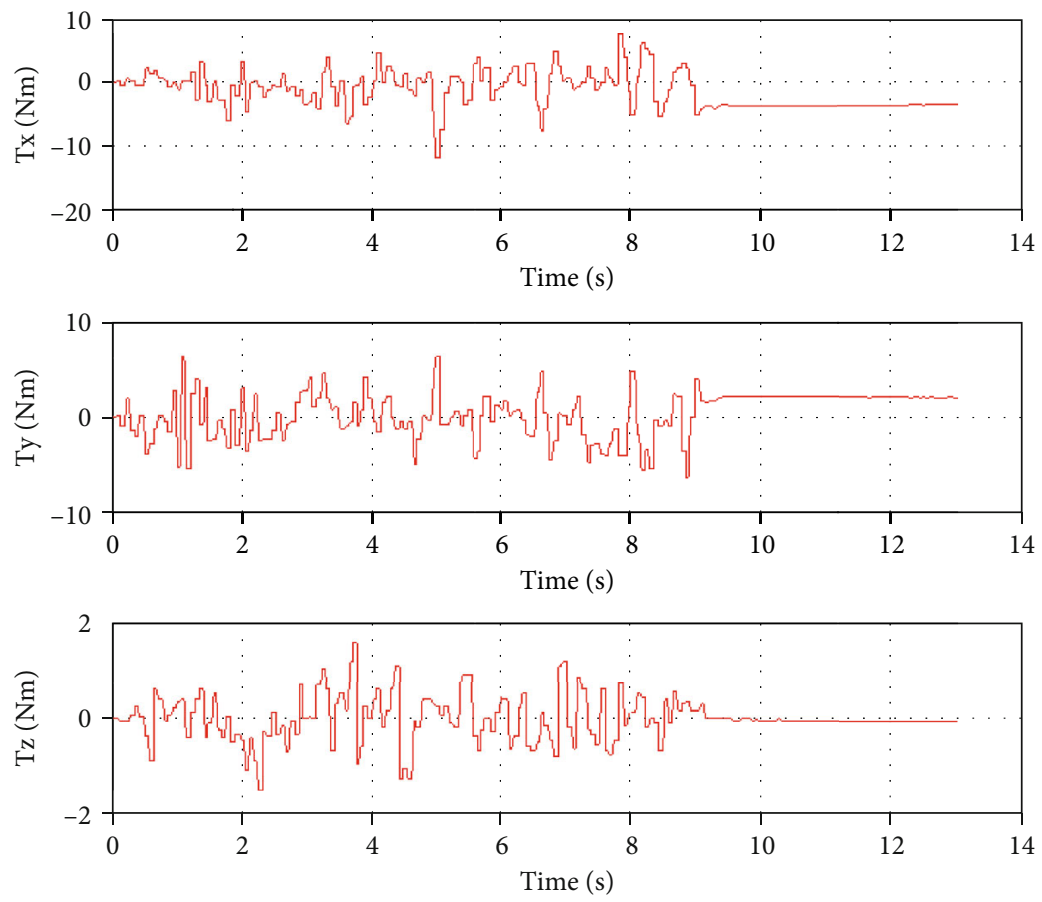

(b)

FIgURE 10: Pure contact force and torque during the inserting stage: (a) force; (b) torque.

simulate the vibration of the real CM manipulator, and the simulated EM manipulator end effector is fully docked with the payload's adapter, but it is not locked (Figure 13(a)). This experiment mainly simulates processes of the simulated EM manipulator end effector locking the payload, two arm closed chain movement, simulated CM manipulator end effector unlocking the payload, and EM manipulator pulling out the payload.
Throughout the experiment, the CM manipulator is in position control mode, and its end moves in the XY plane in a triangular trajectory with a speed of $10 \mathrm{~mm} / \mathrm{s}$ to simulate vibration. When the experiment starts, the simulated EM manipulator end effector electromagnet is energized immediately to lock the payload adapter (Figure 13(a)). The EM manipulator is in a control state of docking, i.e., in position control mode in the $\mathrm{Z}$ direction, in compliant control mode 

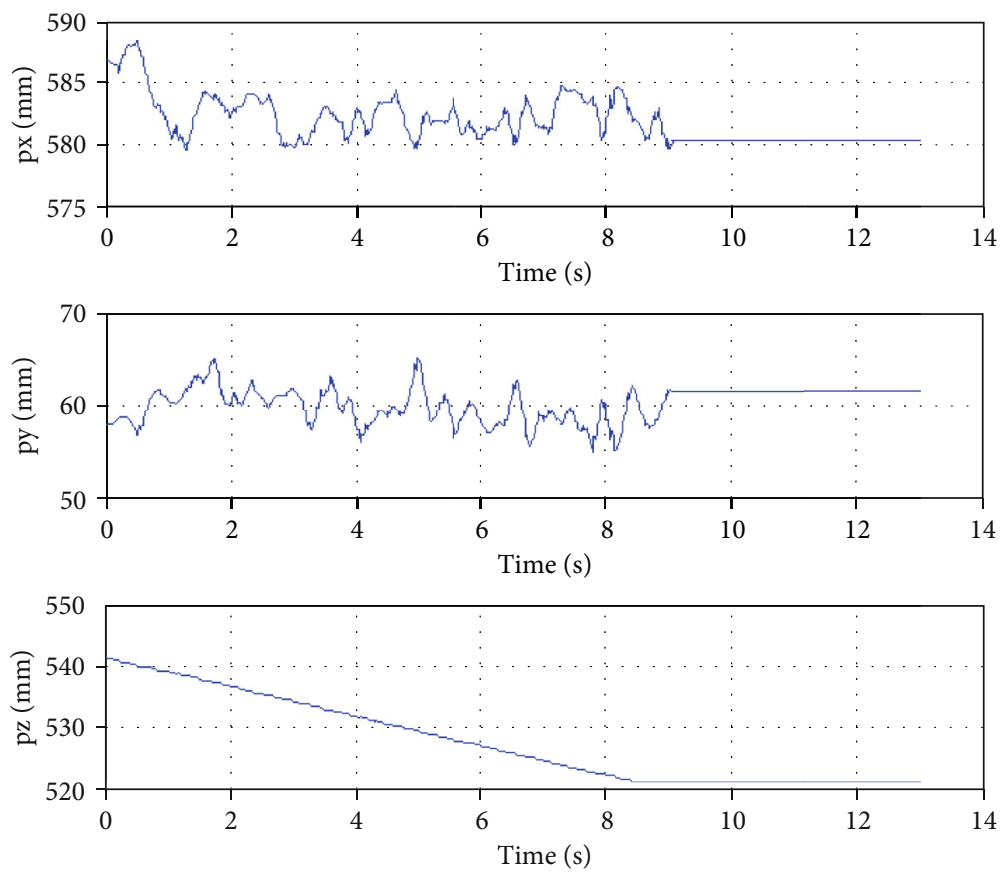

(a)


(b)

FIgURE 11: Real-time end pose of the simulated EM manipulator during the inserting stage: (a) position; (b) attitude.

in the other directions. In Figure 13(b), the two arm system is in the close chain motion stage, and the simulated EM manipulator is in compliance control mode in all directions. In Figure 13(c), the CM manipulator end effector releases the payload, while the EM manipulator with the payload transfers along the $Z$ axis gradually away from the CM manipulator end effector; the other directions of the EM manipulator are in compliance control mode. Figure 13(d) shows that the EM manipulator with the payload separates with the
CM manipulator successfully, and the EM manipulator is ready to move into position control mode to transport the payload to another position in a free space.

Figure 14 is the pure contact force and torque obtained by the force/torque sensor mounted at the end of the simulated EM manipulator. Figure 15 is the real-time end pose of the simulated EM manipulator. The dashed lines in Figures 14 and 15 represent the state change of the simulated EM manipulator. In Figure 14, from $0 \mathrm{~s}$ to $7 \mathrm{~s}$, because the EM 


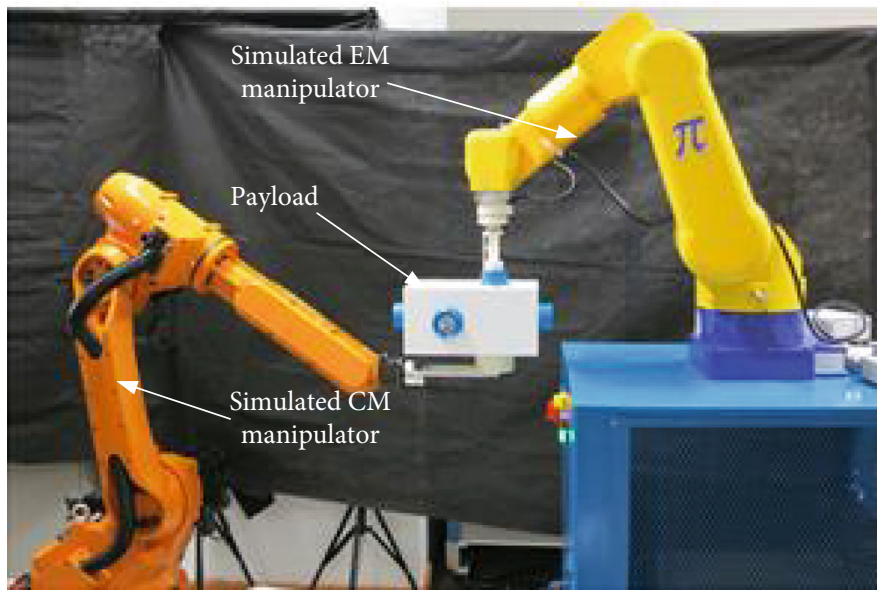

FIGURE 12: Dual arm collaborative payload transport experiment.

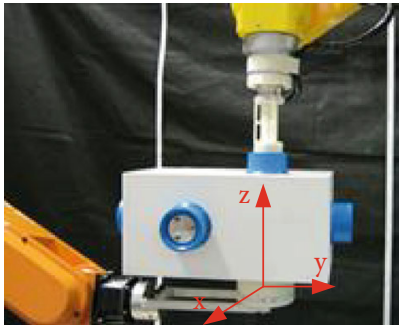

(a)

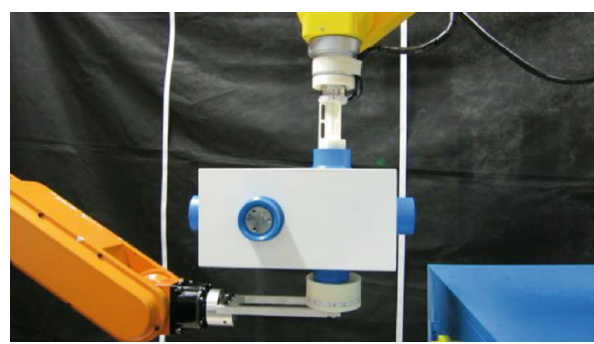

(c)

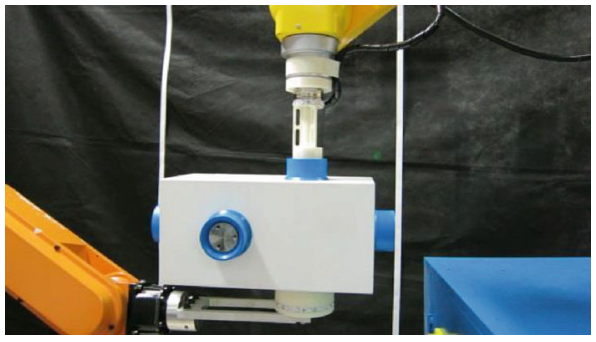

(b)

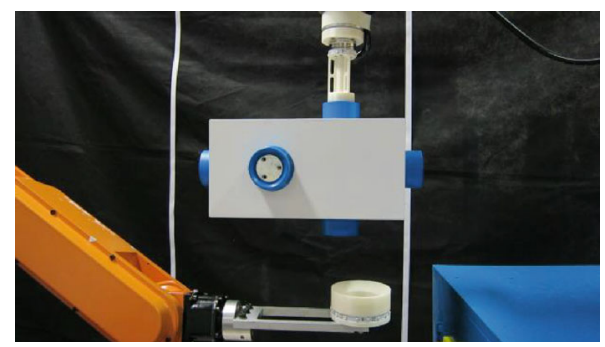

(d)

FIGURE 13: Dual arm collaborative payload transport experiment: (a) initial state; (b) docking state; (c) close chain motion; (d) pulling out state.

manipulator is in docking stage, the contact force of the simulated EM manipulator in the $Z$ axis is larger. In other directions, due to the influence of compliance control, the simulated EM manipulator follows the simulated CM manipulator under contact force/torque, and the contact force/torque remains oscillating near 0 . From $7 \mathrm{~s}$ to $13 \mathrm{~s}$, the manipulator system is in close chain motion stage, and the simulated EM manipulator is in compliance control mode in all axes. The simulated EM manipulator follows the end vibration of the simulated CM manipulator, and the contact force along the $Z$ direction of the simulated EM manipulator tends to decrease, while the rest of the contact force remains near 0 . From $13 \mathrm{~s}$ to $15 \mathrm{~s}$, the CM manipulator effector is unlocked. The position of the EM manipulator in the $Z$ direction increases gradually, and the contact force remains oscillating near 0 in other directions, indicating that the EM manipulator carries the payload away from the CM manipulator effector. Through the above experiments, it is proved that the force compliance control method proposed in Section 3.1 can be used for the dual arm collaborative payload transport, and the contact force/torque can be controlled effectively.

\section{Conclusions}

During the construction and operation stages of the Chinese space station, a large number of orbit tasks with contact force exist. In view of the requirement of ORU replacement and dual arm collaborative payload transport, task flows and requirements of force compliance control are analyzed firstly. Analysis results show that, at the different stages of the above tasks, the EM manipulator should be in force compliance control mode in part or in all directions, and a position based impedance control method in Cartesian space is adopted in this paper to control the contact force/torque. By collecting the contact force/torque at the end of the manipulator using a sensor, the Cartesian space pose adjustment value is 

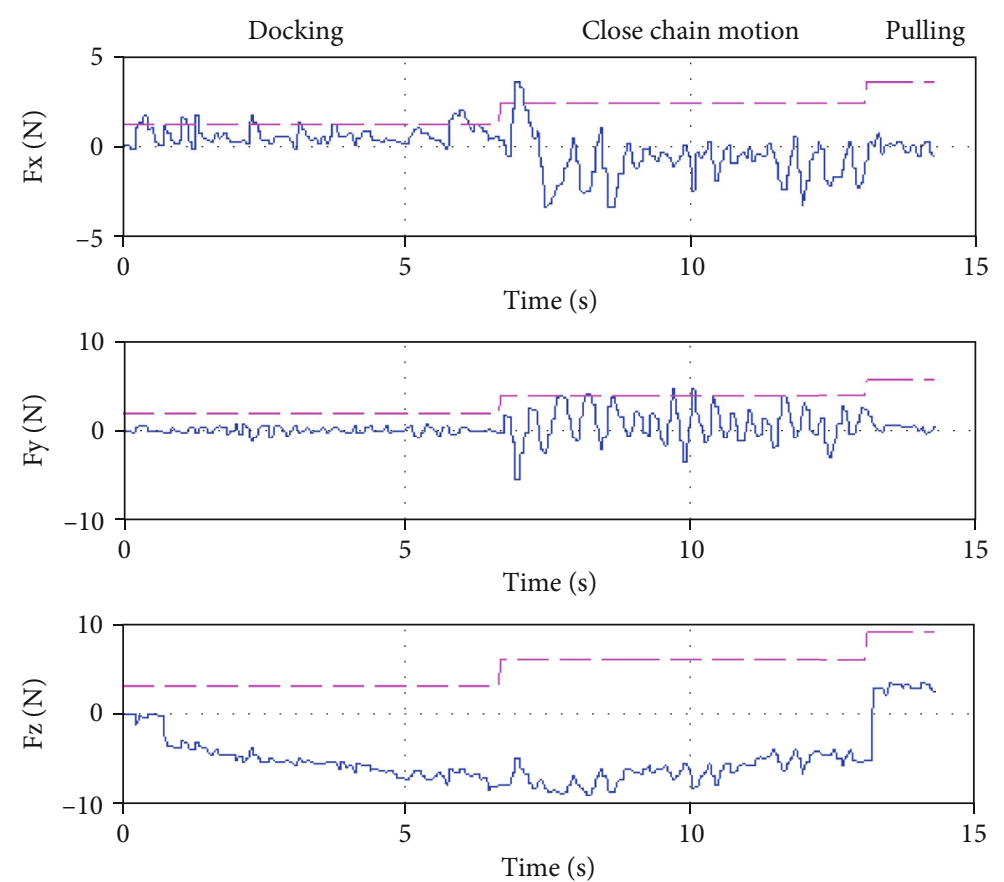

(a)
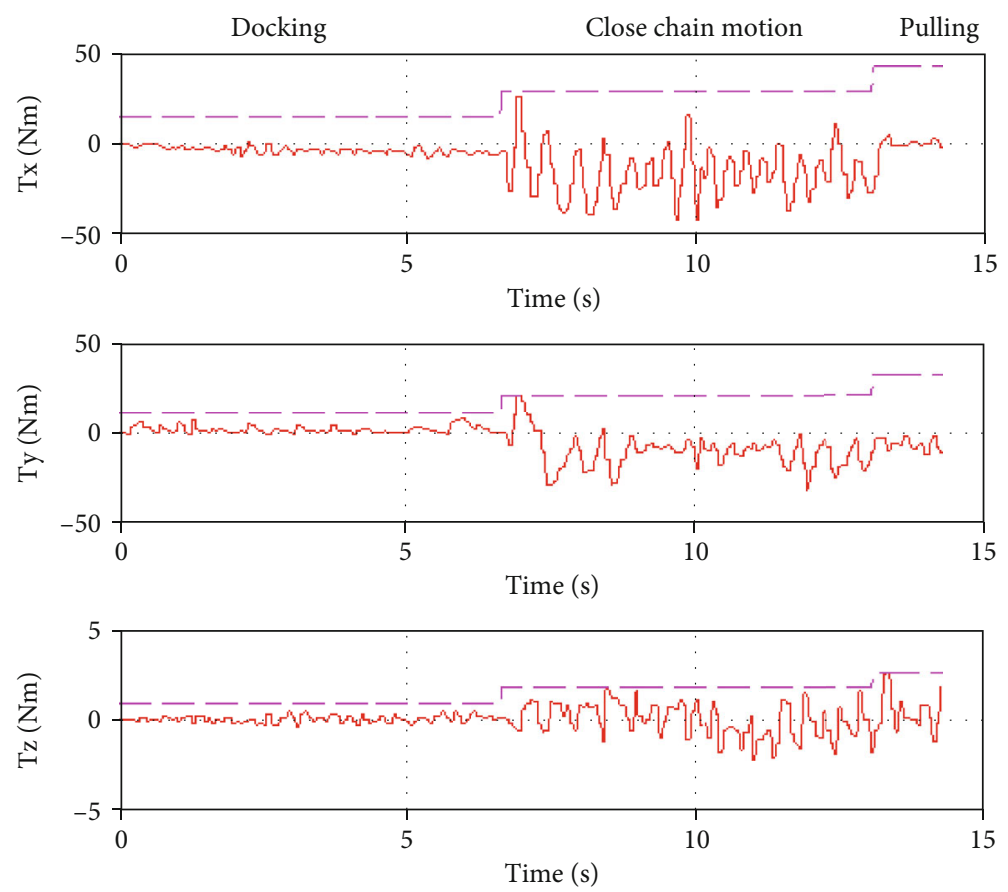

(b)

FIGURE 14: Pure contact force and torque of the simulated EMM during the experiment: (a) force; (b) torque.

obtained according to the desired inertia, damping, and stiffness parameters. For engineering implementation, this paper also analyzes the selection method of key parameters in impedance control and studies the discretization method of the impedance control algorithm. In order to verify the validity of the control algorithm, an experimental platform is built with industrial manipulators. In order to eliminate the influence of gravity, a real-time gravity compensation algorithm is studied to obtain the pure contact force and torque. Using the above experimental platform, the correctness of the real-time gravity compensation and force compliance control algorithm is verified firstly. Then, ORU replacement and dual arm collaborative payload transport are carried out, and the real-time end pose and pure contact force/torque are collected. Experimental results show that the force compliance control method proposed in this paper can control the contact force and torque at the end of the manipulator when performing typical tasks. 



(a)
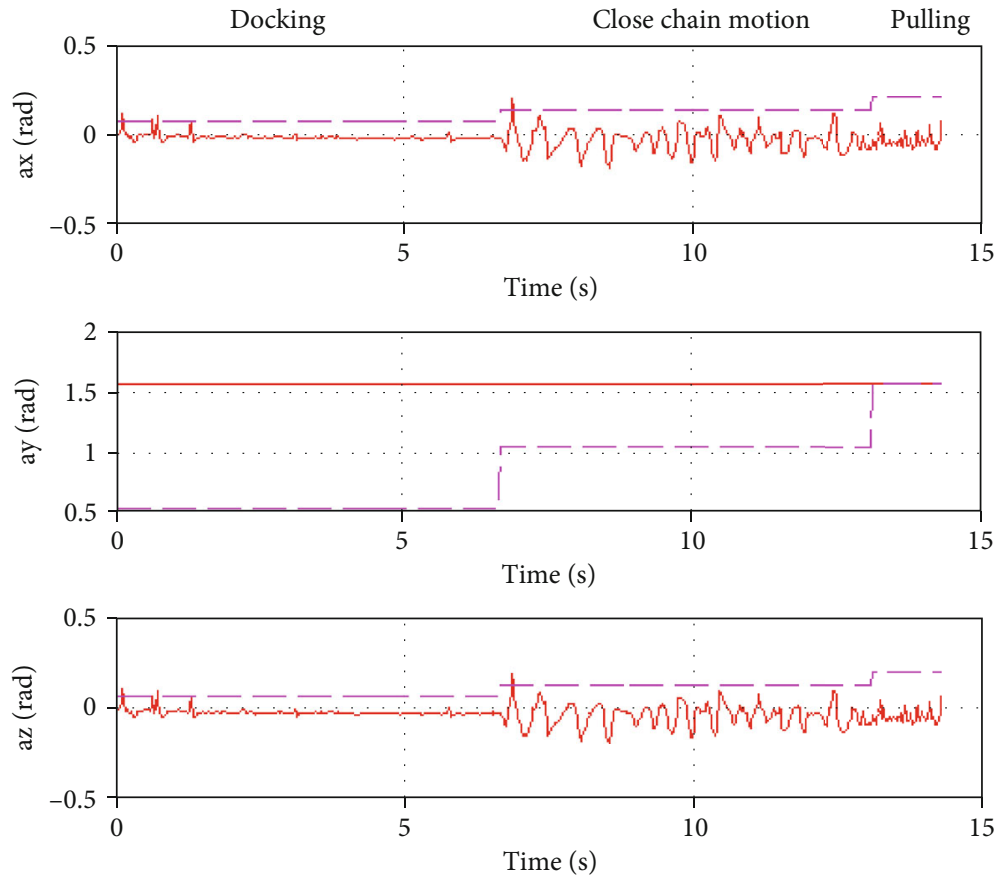

(b)

FIGURE 15: End pose of the simulated EMM during the experiment: (a) position; (b) attitude.

\section{Data Availability}

The data used to support the findings of this study are available from the corresponding author upon request.

\section{Conflicts of Interest}

No potential conflict of interest was reported by the authors.

\section{References}

[1] A. Flores-Abad, O. Ma, K. Pham, and S. Ulrich, "A review of space robotics technologies for on-orbit servicing," Progress in Aerospace Science, vol. 68, pp. 1-26, 2014.

[2] P. Laryssa, E. Lindsay, O. Layi et al., "International space station robotics: a comparative study of ERA, JEMRMS and MSS," in Proceedings of the 7th ESA Workshop on Advanced 
Space Technologies for Robotics and Automation, Noordwijk, Netherlands, November 2002.

[3] Z. Jianping, "Chinese space station project overall vision," Manned Spaceflight, vol. 2, pp. 1-10, 2013.

[4] H. Liu, "An overview of the space robotics progress in China," in Proceedings of the 12th International Symposium on Artificial Intelligence, Robotics and Automation in Space, Montreal, Canada, June 2014.

[5] L. Daming, R. Wei, H. Chengwei, W. Yaobing, T. Zixin, and W. Youyu, "Overview of the Chinese space station manipulator," in Proceedings of the AIAA SPACE 2015 Conference and Exposition, SPACE Conferences and Exposition, Pasadena, CA, USA, August 2015.

[6] C. Ott, R. Mukherjee, and Y. Nakamura, "A hybrid system framework for unified impedance and admittance control," Journal of Intelligent and Robotic Systems, vol. 78, no. 3-4, pp. 359-375, 2015.

[7] N. Hogan, "Impedance control: an approach to manipulation," in 1984 American Control Conference, San Diego, CA, USA, 1984.

[8] H. Nakanishi and K. Yoshida, "Impedance control for freeflying space robots - basic equations and applications," in Proceedings of the IEEE/RSJ International Conference on Intelligent Robots and Systems, Beijing, China, October 2006.

[9] A. Stolfi, M. Sabatini, P. Gasbarri, and G. B. Palmerini, "Analysis of rendezvous maneuvers exploiting impedance control technique," in Proceedings of the 3rd IAA Conference on Dynamics and Control of Space Systems, Moscow, Russia, May 2017.

[10] W. Xu, J. Peng, B. Liang, and Z. Mu, "Hybrid modeling and analysis method for dynamic coupling of space robots," IEEE Transactions on Aerospace and Electronic Systems, vol. 52, no. 1, pp. 85-98, 2016.

[11] A. Ogilvie, J. Allport, M. Hannah, and J. Lymer, "Autonomous robotic operations for on-orbit satellite servicing," in Proceedings of SPIE Sensors and Systems for Space Applications II, Orlando, FL, USA, March 2008.

[12] T. Matsueda, K. Kuraoka, K. Goma, T. Sumi, and R. Okamura, "JEMRMS System Design and Development Status," in NTC '91 - National Telesystems Conference Proceedings, Atlanta, GA, USA, March 1991.

[13] L. Jiang, X. Huo, Y. Liu, and H. Liu, "Optimization-based compliance control strategy of redundant robot for ORU replacements," in 2016 12th World Congress on Intelligent Control and Automation (WCICA), Guilin, China, June 2016.

[14] Y. Mo, S. Gao, Z. Jiang, H. Li, and Q. Huang, "Impedance control with force signal compensation on space manipulator-assisted docking mission," in 2014 IEEE International Conference on Information and Automation (ICIA), Hulun Buir, China, July 2014.

[15] R. Boumans and C. Heemskerk, "The European robotic arm for the international space station," Robotics and Autonomous System, vol. 23, no. 1-2, pp. 17-27, 1998.

[16] B. Hu, M. Chen, H. Zou, H. Chen, and F. Chen, "Control of a manipulator for replacing the orbit replacement unit on the space station," in 2015 IEEE International Conference on Robotics and Biomimetics (ROBIO), Zhuhai, China, December 2015.
[17] C. Barbalata, M. W. Dunnigan, and Y. Petillot, "Coupled and decoupled force/motion controllers for an underwater vehicle-manipulator system," Journal of Marine Science and Engineering, vol. 6, no. 3, p. 96, 2018.

[18] G. Colombina, F. Didot, G. Magnani, and A. Rusconi, "External servicing testbed for automation and robotics," IEEE Robotics and Automation Magazine, vol. 3, no. 1, pp. 13-23, 1996. 